\title{
A Flexible Multiplatform Bioanalytical Strategy for Measurement of Total Circulating Shed Target Receptors: Application to Soluble B Cell Maturation Antigen Levels in the Presence of a Bispecific Antibody Drug
}

\author{
Angela Stauffer, ${ }^{1}$ Chad Ray, ${ }^{2}$ and Michael Hall ${ }^{1}$ \\ ${ }^{1}$ Biomedicine Design, Pfizer Worldwide Research, Development, \\ and Medical, San Diego, California, USA. \\ ${ }^{2}$ Zoetis Incorporated, Fort Collins, Colorado, USA.
}

\begin{abstract}
$B$ cell maturation antigen (BCMA) is a membrane-bound receptor that is overexpressed on multiple myeloma cells and can be targeted with biotherapeutics. Soluble shed forms of membraneassociated receptors in circulation can act as a drug sink, especially when it is present in high molar ratio compared to drug concentration, potentially derailing the intended pharmacological mechanism and impacting pharmacokinetic (PK) measurements and efficacious dose predictions. In this study, we present a bioanalytical strategy for assessing dynamic levels of total soluble BCMA before and during treatment with a bispecific antibody targeting BCMA and $C D 3$. Implementation of a ligand binding assay was not successful due to extensive bispecific antibody interference. Instead, we explored two types of immunoaffinity (IA) liquid chromatography-tandem mass spectrometry (LC-MS/MS) assays, one at the protein level and one at the surrogate peptide level. Ultimately, the protein-level IA-LC-MS/MS method was optimized for use in a cynomolgus monkey PK/pharmacodynamic study. In addition, we demonstrated that the method was easily adapted for use with human samples in preparation for translation to the clinic. This work demonstrates the benefit of flexibility and agility in bioanalytical method development in early drug development. Multiplatform suitability assessments enable rapid, resourcesparing identification and qualification of clinically translatable assays. We recommend early adoption of this strategy to provide enough time for critical reagent development and assay validation for analysis of shed targets.
\end{abstract}

Keywords: shed target, bioanalysis, method development, drug interference, BCMA

\section{INTRODUCTION}

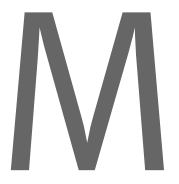

any biotherapeutic drugs are designed to bind to
membrane-bound cellular receptors to elicit a
desired pharmacological effect. In some cases,
the extracellular domain of these receptors may be released from the cell surface through a regulated proteolytic mechanism called ectodomain shedding. ${ }^{1,2}$ Shed target receptors can act as a neutralizing sink that reduces drug binding to the membrane-bound target and thereby reduces the efficacy of the biotherapeutic., If this interaction is not taken into consideration, then pharmacokinetic (PK) measurements, including drug exposure and clearance, may be inaccurate, leading to miscalculation of the predicted efficacious dose. Ectodomain shedding can vary throughout disease progression and after drug treatment, thereby changing the size of the neutralizing sink and further complicating PK measurements and the overall translational strategy. Determination of shed receptor levels may also be useful as a pharmacodynamic (PD) biomarker in that changes in shedding after drug treatment may be correlated with pharmacological efficacy. ${ }^{1,5}$

B cell maturation antigen (BCMA) CD3 bispecific antibodies have one binding domain targeting BCMA, which is overexpressed on malignant multiple myeloma (MM) plasma cells, and one domain that binds CD3 on T cells (Fig. 1A). The intended mechanism of action occurs when the bispecific antibody brings plasma tumor cells and T cells into proximity, allowing for the formation of an immunological synapse and $\mathrm{T}$ cell mediated destruction of the tumor cell. Elevated levels of soluble BCMA (sBCMA) have been reported in MM patients, and the degree of elevation correlates with disease 


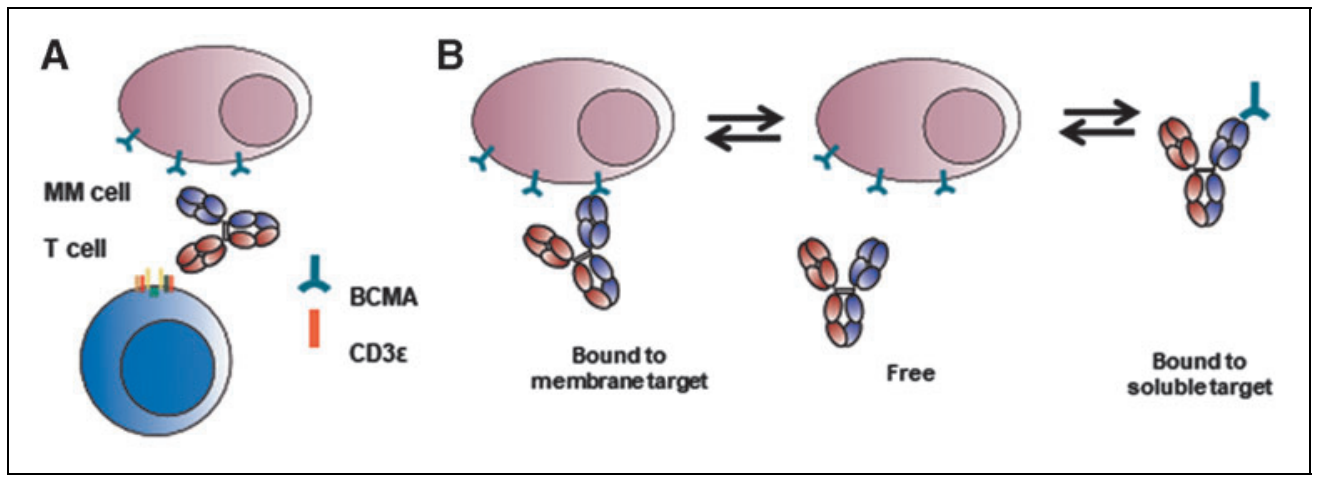

Fig. 1. Mechanism of drug action and impact of $s B C M A$. (A) $B C M A C D_{3}$ bispecific antibody binds to membrane-bound BCMA on MM cells and $C_{3}$ on T cells. (B) sBCMA in the central compartment can compete with membrane-bound BCMA for binding to the drug, thereby producing a sink that can reduce the efficacious drug concentration. Levels of the soluble form may also be used as a biomarker for disease progression. BCMA, B cell maturation antigen; MM, multiple myeloma; sBCMA, soluble BCMA.

progression. ${ }^{6}$ It has also been demonstrated that BCMA is specifically shed by $\gamma$-secretase, an intramembrane protease. ${ }^{7}$ High levels of sBCMA in circulation can potentially hinder the intended pharmacological mechanism of BCMA CD3 bispecific antibodies (Fig. 1B). Therefore, it is crucial to ascertain treatment-induced changes in sBCMA to assess potential impacts on PK. Understanding the levels of shed target is even more critical for bispecific antibodies, since toxicities associated with dose escalation are not necessarily well understood.

Given the potential impact of shed target concentrations, bioanalytical methods should be considered early in the development of the translational plan. In this article, we present a flexible multiplatform bioanalytical strategy for assessing total sBCMA levels in the presence or absence of BCMA CD3 bispecific antibody. The total sBCMA measurement, as opposed to free or partially bound measurements, was determined to be the best fit-for-purpose measurement to support early modeling and predictive dosing simulation efforts. Total target measurements are often preferred in the preclinical stage due to technical challenges of free target measurements, including reagent availability, assay sensitivity, and the dynamic equilibrium between the drug and its target. ${ }^{8,9} \mathrm{In}$ some cases, the total ligand measurements can be used to infer the free ligand concentrations using a mechanistic PK/PD model. ${ }^{3}$ It is important to reevaluate the type of shed target assay used as the drug program progresses as the needs may change once the project reaches a later stage or new reagents become available.

Ligand binding assays (LBAs) are often the preferred bioanalytical platform for measuring shed targets due to multiple factors, including the availability of commercial re- agents, ease of use, availability of automation, and widespread adoption at contract research organizations. Since we intended to monitor sBCMA levels after biotherapeutic drug treatment in cynomolgus monkeys and we hypothesized that free levels would become vanishingly small after dosing, it was imperative to consider drug interference during development of the total sBCMA assay. Our initial LBA format suffered from drug interference at even low levels of drug, and although we could have considered further efforts to salvage an LBA approach, such as implementation of an acid dissociation step, ${ }^{10,11}$ we decided to turn our attention to alternative bioanalytical platforms. Specifically, liquid chromatographytandem mass spectrometry (LC-MS/MS) methods are an increasingly popular platform for quantifying proteins in biological samples. Many of these methods have sensitivities that are comparable to those of LBA, especially when an initial immunoaffinity (IA) step is used to enrich for the target of interest before processing for MS analysis. ${ }^{12,13}$ Since antibody reagents may compete with drug as shown during our LBA assessment, we considered both protein-level and peptide-level IA-LC-MS/MS assay formats. Ultimately, the protein-level IA-LC-MS/MS method was selected as the assay format to be optimized for preclinical use in cynomolgus monkeys and was eventually converted to an assay that could be used for human patient samples.

Fit-for-purpose bioanalytical qualification typically involves specific analytical platforms or technical approaches (e.g., LBA) through later stage validation. This report describes a flexible multiplatform assay assessment strategy that incorporates multiple bioanalytical platforms and assay formats to identify the best fit-for-purpose assay quickly. This strategy for bioanalysis of total sBCMA levels can be more widely applied to any biotherapeutic where shed receptor levels must be measured and demonstrates the advantages of having a diverse suite of bioanalytical assays. Drug interference should be considered early in method development with available LBA formats and reagents. If available resources are not suitable, then development of new reagents for use in either LBA or alternate platforms such as LC-MS/MS needs to commence in a timely manner. 


\section{MATERIALS AND METHODS}

\section{Reagents and Materials}

Bispecific antibodies targeting BCMA and CD3 were generated at Pfizer and expressed in HEK293 cells. The extracellular domains of both cynomolgus monkey and human BCMA were cloned into a proprietary in-house recombinant mammalian expression vector with a C-terminal $10 \times$ His and AviTag $^{\text {TM }}$ (Avidity, LLC, Aurora, CO) and expressed in HEK293 cells. Human plasma samples from patients with late stage MM were obtained from Cureline (Brisbane, CA). All deidentified human plasma samples provided for this project were collected from consented patients in collaboration with major research/clinical centers under the local Ethics Committee/Institutional Review Board approved protocols. All reagents and solvents were reagent grade unless otherwise noted.

\section{Animal Use and Care}

This study was conducted in accordance with the current guidelines for animal welfare (National Research Council Guide for the Care and Use of Laboratory Animals, 2011; Animal Welfare Act [AWA], 1966, as amended in 1970, 1976, 1985, and 1990, and the AWA implementing regulations in Title 9, Code of Federal Regulations, Chapter 1, Subchapter A, Parts 1-3). The procedures used in this study were reviewed and approved by the Institutional Animal Care and Use Committee.

The cynomolgus monkey study was conducted at Pfizer (Groton, CT). Monkeys were housed in same-sex pairs in stainless steel cages and were provided environmental enrichment. Monkeys were fed a certified primate diet (5K91, PMI Feeds, Richmond, IN) daily in amounts appropriate for the age and size of the animals and had ad libitum access to municipal water, further purified by reverse osmosis. Monkeys were maintained on a 12-h light/12-h dark cycle in rooms with temperature maintained between $66^{\circ} \mathrm{F}$ and $77^{\circ} \mathrm{F}$ and humidity between 30\% and 70\%.

Female cynomolgus monkeys ( $>2.5$ years old) received a single IV bolus dose of BCMA bispecific antibody (1, 10, or $100 \mu \mathrm{g} / \mathrm{kg}$ ). Plasma was periodically collected through 7 days postdose.

\section{LBA for sBCMA}

Human BCMA/TNFRSF17 DuoSet ${ }^{\circledR}$ Enzyme-Linked Immunosorbent Assay (ELISA) Kit from RetD Systems (DY193, Minneapolis, MN) was converted to a Meso Scale Discovery ${ }^{\circledR}$ (MSD, Rockville, MD) format and evaluated for crossreactivity toward cynomolgus monkey BCMA and suitability for use in the presence of BCMA CD3 bispecific antibody. Manufacturer's instructions were followed, except where in- dicated. Super Block (ScyTek, Logan, UT) was used as the assay diluent and blocking buffer. Wash Solution Concentrate containing $20 \times$ imidazole-buffered saline with Tween ${ }^{\circledR} 20$ (SeraCare, Milford, MA) was diluted to $1 \times$ with water and used as the wash buffer. Each well of a high bind SECTOR ${ }^{\circledR}$ MSD plate was coated with $50 \mu \mathrm{L}$ of $1 \mu \mathrm{g} / \mathrm{mL}$ goat anti-human BCMA capture antibody from the DuoSet ELISA Kit. Standards and quality controls (QCs) were prepared by serially diluting recombinant cynomolgus monkey BCMA in Super Block. QCs spiked with BCMA CD3 bispecific antibody were incubated for $3 \mathrm{~h}$ at room temperature on a plate shaker. Fifty microliters of each standard and QC were added to the plate in duplicate. SULFO-TAG ${ }^{\circledR}$ labeled streptavidin (MSD) was used to measure the biotinylated anti-BCMA detection antibody from the RAD Kit, and Read Buffer T (MSD) diluted to $2 \times$ with water was added to each well before measuring the electrochemiluminescent signal $(620 \mathrm{~nm})$ on the MESO ${ }^{\circledR}$ SECTOR S 600 instrument. Regression was performed on the MSD DISCOVERY WORKBENCH ${ }^{\circledR}$ v4.0 software using 4PL $1 / \mathrm{y}^{2}$ weighting. QCs were backfit to the standard curve to determine \% recovery.

\section{Selection of Surrogate sBCMA Peptide for LC-MS/MS Analysis}

An AB Sciex QTRAP ${ }^{\circledR} 6500$ mass spectrometer running in positive-ion multiple reaction monitoring (MRM) mode coupled with a Shimadzu Prominence UFLC ${ }^{\circledR}$ LC-20AD XR liquid chromatography interface was used for all LC-MS/MS studies. Potential surrogate peptides of sBCMA were identified using Skyline software (MacCoss Lab; University of Washington, Seattle, WA). A default MRM method was generated within Skyline. MRM transitions and collision energies were further optimized empirically by digesting recombinant cynomolgus monkey sBCMA and analyzing the peptide digest by LC-MS/MS. Only one sBCMA surrogate peptide was found to have desirable chromatographic retention time, ionization efficiency, and $\mathrm{MS}^{2}$ fragmentation: CSSTPPLTCQR from the cynomolgus monkey protein and CSSNTPPLTCQR in the homologous human protein. Both sequences were searched using Basic Local Alignment Search Tool (BLAST ${ }^{\circledR}$ ) and were confirmed to be unique within each organism. MRM transitions used for quantification of cynomolgus monkey and human sBCMA peptides were $m / z 653.8 \rightarrow 871.4$ and $m / z$ $710.8 \rightarrow 871.4$, respectively.

\section{Preparation of sBCMA Heavy-Isotope Labeled Peptide} Internal Standard

Heavy-isotope labeled peptide internal standard (IS) was generated by diluting recombinant cynomolgus monkey 
sBCMA (20 $\mu \mathrm{g}$ ) into $190 \mu \mathrm{L} 158 \mathrm{mM}$ TrisHCl, pH 8. To enhance enzymatic digestion, RapiGest ${ }^{\mathrm{TM}}$ SF surfactant (Waters, Milford, MA) was added to the reaction mixture $(10 \mu \mathrm{L} 2.4 \%$ aq. [w/v] solution). Disulfides were reduced by addition of $15 \mu \mathrm{L}$ $75 \mathrm{mM}$ aqueous Tris(2-carboxyethyl)phosphine (TCEP; Thermo Scientific, Rockford, IL) and incubating for $45 \mathrm{~min}$ at $60^{\circ} \mathrm{C}$. Alkylation of free thiols with introduction of heavy isotopes was achieved by addition of $20 \mu \mathrm{L} 75 \mathrm{mM}$ labeled iodoacetamide, $\mathrm{I}^{13} \mathrm{CD}_{2}{ }^{13} \mathrm{C}(0) \mathrm{NH}_{2}$ (Sigma-Aldrich, 721328-50MG, 98\% atom $\mathrm{D}, 99 \%$ atom ${ }^{13} \mathrm{C}$ ) and incubating for $1 \mathrm{~h}$, in the dark, at room temperature. The labeled sBCMA was then digested by addition $(20 \mu \mathrm{L})$ of an aqueous trypsin/Lys-C enzyme (mass spectrometry grade; Promega, Madison, WI) solution (100 ng/ $\mu \mathrm{L}$ ) and incubating at $37^{\circ} \mathrm{C}$, in the dark, overnight. The sample was then acidified with $1.3 \mu \mathrm{L}$ neat Trifluoroacetic acid (TFA; ProteoChem, Hurricane, UT) and heated for $45 \mathrm{~min}$ at $37^{\circ} \mathrm{C}$. Precipitated RapiGest SF was removed by centrifugation (20 min, 14,000 g, room temperature). Without further purification, small aliquots $(5 \mu \mathrm{L})$ of IS were stored at $-80^{\circ} \mathrm{C}$. The selected reaction monitoring transition $\mathrm{m} / \mathrm{z} \quad 657.8 \rightarrow 875.5$ was utilized for monitoring the IS. IS was added to each sample such that the extracted ion chromatogram (XIC) peak area was within 10\%-100\% of the XIC peak areas of sBCMA surrogate peptide in analyzed samples. This IS was also used for analysis of human samples.

\section{Generation of Polyclonal Antibodies Against sBCMA \\ Surrogate Peptide}

Polyclonal antibodies were generated in rabbits against the surrogate cynomolgus monkey sBCMA peptide CSSTPPLTCQR (Cambridge Research Biochemicals, Billingham, UK). Due to peptide chemistry limitations, serine was substituted for the second carbamidomethylated cysteine in the peptide used for immunization (CSSTPPLTSQR). After conjugation of the peptide to keyhole limpet hemocyanin (KLH), rabbits were injected with the peptide-KLH complex. Antisera were screened for antibodies that bound to the peptide immunogen and the corresponding human peptide (CSSNTPPLTCQR) to ensure cross-reactivity, followed by affinity purification against the peptide immunogen.

\section{Biotinylation of Antibodies Used for IA Enrichment}

Polyclonal goat anti-human BCMA antibody (RED Systems) and polyclonal rabbit anti-peptide (CSSTPPLTCQR) antibodies (Cambridge Research Biochemicals) were buffer exchanged into phosphate-buffered saline (PBS), pH 7.4 (Gibco, Grand Island, NY) using a Zeba ${ }^{\mathrm{TM}}$ spin desalting column, $40 \mathrm{k}$ MWCO (Thermo Scientific) following the manufacturer's instructions. Buffer-exchanged antibodies were conjugated with biotin at a challenge ratio of 12:1 (biotin:antibody) using EZ-Link ${ }^{\mathrm{TM}}$ Sulfo-NHS-LC-Biotin (Thermo Scientific).

\section{Protein-Level IA-LC-MS/MS Sample Preparation}

A standard curve was prepared by spiking recombinant cynomolgus or human BCMA into filtered rat plasma (K2 EDTA; BioIVT, Hicksville, NY) followed by a serial dilution. QCs were prepared in rat plasma during method development and in cynomolgus plasma when testing cynomolgus samples with and without BCMA CD3 bispecific antibody. Biotinylated goat polyclonal anti-BCMA (DY193; R\&D Systems) was added to each standard, QC, and samples followed by fourfold dilution in Super Block and then incubated at $4^{\circ} \mathrm{C}$ overnight on a rotator.

Prewashed streptavidin magnetic beads $(140 \mu \mathrm{L}$, Dynabeads $^{\mathrm{TM}} \mathrm{MyOne}^{\mathrm{TM}}$ Streptavidin $\mathrm{C} 1$; Invitrogen, Norway) were added to each sample and incubated at room temperature on a rotator for $1 \mathrm{~h}$. Beads were washed twice with 0.05\% aq. (w/v) 3-[(3-cholamidopropyl)dimethylammonio]1-propanesulfonate (CHAPS; Sigma, St. Louis, MO) in PBS, then and once with PBS alone. One hundred forty microliters of aqueous hydrochloric acid $(\mathrm{HCl}, 25 \mathrm{mM})$ was added to elute the antibody from the beads. Samples were incubated at room temperature for $30 \mathrm{~min}$ and mixed briefly on a vortexer every $10 \mathrm{~min}$. Eluates were transferred to fresh microcentrifuge tubes and neutralized with $30 \mu \mathrm{L}$ of $1 \mathrm{M}$ Tris $\mathrm{HCl}, \mathrm{pH}$ 8.0. RapiGest SF surfactant ( $10 \mu \mathrm{L}$ of $2.2 \%$ aq. [w/v] solution) was added to each sample, and reduction was achieved by addition of $15 \mu \mathrm{L} 75 \mathrm{mM}$ aq. TCEP and incubating at $60^{\circ} \mathrm{C}$ for $45 \mathrm{~min}$. Alkylation of reduced thiols proceeded by addition of $15 \mu \mathrm{L}$ of $150 \mathrm{mM}$ iodoacetamide and incubating for $1 \mathrm{~h}$ in the dark at room temperature. Samples were digested by addition of $10 \mu \mathrm{L}$ of $100 \mathrm{ng} / \mathrm{mL}$ trypsin/Lys-C and incubating at $37^{\circ} \mathrm{C}$ overnight with shaking as above. Neat TFA $(1.1 \mu \mathrm{L})$ was added, and samples were incubated at $37^{\circ} \mathrm{C}$ for $45 \mathrm{~min}$. Heavyisotope labeled IS ( $5 \mu \mathrm{L})$ was added to each sample. Samples were centrifuged at room temperature for $10 \mathrm{~min}, 16,000 \mathrm{~g}$, and supernatants were transferred to a Protein LoBind ${ }^{\circledR}$ 96deepwell plate (Eppendorf, Hamburg, GE).

\section{Peptide-Level IA-LC-MS/MS Sample Preparation}

The sample preparation method was adapted from that of Razavi et al. ${ }^{14}$ A denaturation mix was prepared by dissolving urea, Bond-Breaker ${ }^{\mathrm{TM}}$ TCEP solution (Thermo-Fisher, Rockford, IL), and Trizma ${ }^{\circledR}$ pre-set crystals, $\mathrm{pH} 8.1$ in water to yield final concentrations of 9, 0.05, and $0.2 \mathrm{M}$, respectively. One hundred seventy microliters of the "denaturation mix" was aliquoted into a 96-deepwell plate, and the mixture was dried 


\section{STRATEGY FOR BIOANALYSIS OF SHED TARGET RECEPTORS}

using a SPE DRY-96 vacuum (Argonaut Technologies, Redwood, CA). Cynomolgus monkey plasma spiked with varying levels of recombinant sBCMA with or without BCMA CD3 bispecific antibody was added to each well containing the dried denaturation mix followed by a 30-min incubation at room temperature with vigorous mixing on a plate shaker. Iodoacetamide ( $40 \mu \mathrm{L}, 0.25 \mathrm{M}$ ) was added to the solution followed by incubation for $30 \mathrm{~min}$ on the plate shaker at room temperature protected from light. Finally, the reaction mixture was diluted 1:9 with $0.2 \mathrm{M}$ Trizma buffer $\mathrm{pH} 8.1$ to reach a final urea concentration of $1 \mathrm{M}$. TPCK-treated trypsin $(20 \mu \mathrm{L}$, $37.5 \mathrm{mg} / \mathrm{mL}$; Worthington, Lakewood, NJ) was added to each well. The samples were incubated for $2 \mathrm{~h}$ at $37^{\circ} \mathrm{C}$, followed by addition of $10 \mu \mathrm{L}$ of $2 \mathrm{mg} / \mathrm{mL}$ Tosyl-L-lysine chloromethyl ketone (Sigma).

Each sample was transferred to a $5 \mathrm{~mL}$ microcentrifuge tube and diluted twofold in PBS with 0.01\% CHAPS. Biotinylated antipeptide antibodies (100 $\mu \mathrm{g})$ were added to each sample and incubated at $4{ }^{\circ} \mathrm{C}$ with shaking overnight. Streptavidin-coated magnetic beads $(500 \mu \mathrm{L})$ were used to capture the antibodypeptide complex from solution by incubating on a plate shaker for $1 \mathrm{~h}$. The antibody-coupled beads were collected using the DynaMag ${ }^{\mathrm{TM}}$ - 15 magnet (Invitrogen) and washed twice in PBS/ $0.01 \%$ CHAPS followed by one wash in PBS. The peptides were eluted with $100 \mu \mathrm{L}$ of $25 \mathrm{mM} \mathrm{HCl}$ for 30 min with vortexing every $10 \mathrm{~min}$. Tris- $\mathrm{HCl} \mathrm{pH} 8(30 \mu \mathrm{L}, 1 \mathrm{M})$ was added to each sample for neutralization. TFA $(1.1 \mu \mathrm{L})$ was added to each sample followed by $5 \mu \mathrm{L}$ of heavy-isotope labeled IS.

\section{LC-MS/MS Analysis}

Aliquots of each sample $(25 \mu \mathrm{L})$ were injected and separated on a Kinetex ${ }^{\circledR} \mathrm{C} 18,100 \mathrm{~mm} \times 2.1 \mathrm{~mm}, 2.6 \mu \mathrm{m}, 100 \AA$ column (Phenomenex, Torrance, CA) with a flow rate of $0.2 \mathrm{~mL} / \mathrm{min}$ at $50^{\circ} \mathrm{C}$. Water and acetonitrile (Optima LCMS grade) were both purchased from Fisher Chemical (Fair Lawn, NJ). Formic acid (FA) 99+\% was purchased from Thermo Scientific. The target peptides were separated using an 18 min gradient with $0.1 \%$ aq. FA as solvent $\mathrm{A}$ and $0.1 \% \mathrm{FA}$ in acetonitrile as solvent $\mathrm{B}$. The gradient profile was as follows: $2 \%$ B for 2 min; linear gradient of 2\%-25\% B over $10 \mathrm{~min}$; linear gradient of 25\%80\% B over $2 \mathrm{~min}$; 80\% B for $2 \mathrm{~min}$; linear gradient $80 \%-2 \%$ B over $2 \mathrm{~min}$; $2 \%$ B for $2 \mathrm{~min}$ for column re-equilibration. To prevent any possible carryover, a water blank was injected under the same conditions between samples.

Mass spectrometric acquisition was achieved by accumulating signal (dwell time $100 \mathrm{msec}$, unit resolution) for the MRM transitions $m / z \quad 653.8 \rightarrow 871.4,710.8 \rightarrow 871.4$, and $657.8 \rightarrow 875.5$ corresponding to sBCMA cynomolgus surrogate peptide, human surrogate peptide, and heavy-isotope labeled IS, respectively. XIC peak areas were generated using Analyst 1.6.2, and peak area ratios (PARs) were calculated by dividing the sample XIC peak area by that of the IS. The PARs of the standards were regressed with GraphPad Prism v7.04 using five-parameter logistic regression with $1 / \mathrm{y}^{2}$ weighting. QC and sample concentrations were interpolated by backfitting to the standard curve.

\section{RESULTS}

\section{Assay Development}

To develop the best fit-for-purpose assay to measure total sBCMA plasma levels in the presence of BCMA CD3 bispecific antibody, three separate assay formats were explored: (1) commercial ELISA kit, (2) protein-level IA-LC-MS/MS, and (3) peptide-level IA-LC-MS/MS. The optimal assay would have adequate sensitivity and dynamic range for both preclinical cynomolgus monkey studies and could be transferred to a regulated laboratory and validated more thoroughly to support clinical trials. A previous report ${ }^{6}$ found a range of sBCMA levels from a mean value of $\sim 0.42 \mathrm{nM}$ in normal human plasma to over $83.3 \mathrm{nM}$ in untreated MM patient plasma. This range was used as guidance for assay development in cynomolgus monkey samples. Furthermore, the total sBCMA level measurement needed to be quantitative in the presence of circulating BCMA CD3 bispecific antibody, which required characterization of assay performance and recovery in drug interference experiments.

\section{Modified Commercial ELISA Kit}

Several reports ${ }^{6,15,16}$ utilized a commercial ELISA Kit to measure sBCMA levels. We purchased this kit and converted it to an MSD assay using streptavidin-SULFO-TAG as the detection reagent, to conserve reagents and decrease assay time. We first confirmed that the kit was able to quantitatively measure cynomolgus monkey BCMA by preparing standards and QC samples using recombinant cynomolgus monkey BCMA and ensuring that QC recovery was within an acceptable range. The modified kit was then tested for drug interference by preparing QCs with or without BCMA CD3 bispecific antibody. The BCMA QC levels spanned concentration ranges previously reported for normal and MM patients, while the bispecific concentrations were intended to reflect the range of expected plasma concentrations in preclinical PK studies. In all cases, the recovered sBCMA concentrations fell as BCMA CD3 bispecific antibody concentrations increased (Fig. 2A), indicating that the reagents in the DuoSet Kit and the BCMA targeting arm bind to a shared epitope. Cynomolgus monkey plasma from four treatment naive animals was also spiked with bispecific, yielding similar 

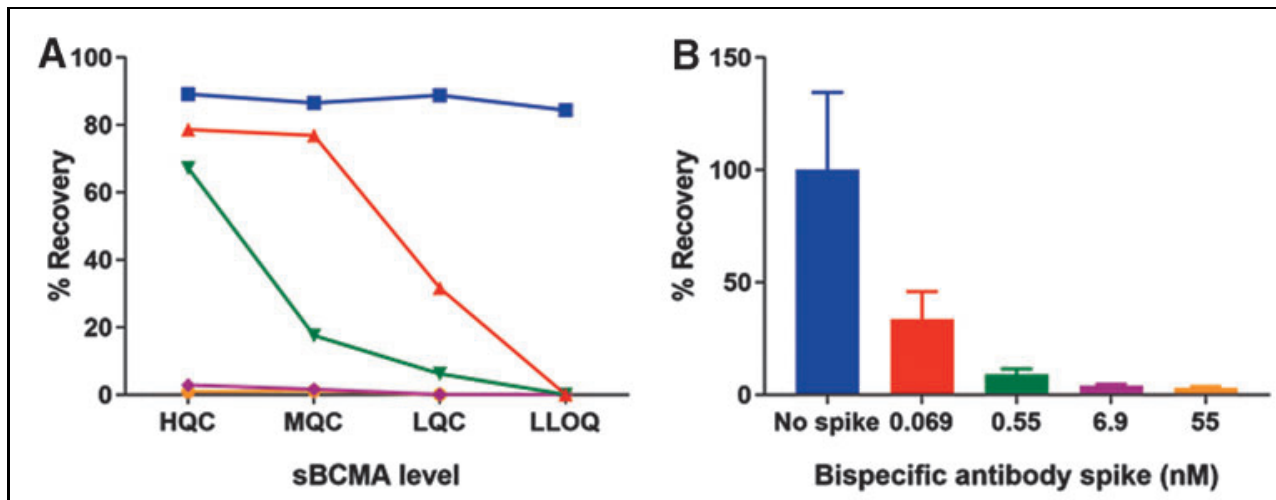

Fig. 2. $B C M A C D_{3}$ bispecific antibody interferes extensively with measurement of total sBCMA by LBA. Four concentrations of $\mathrm{BCMA} \mathrm{CD} 3$ bispecific antibody (blue squares $=$ no spike, red triangle $=$ $0.069 \mathrm{nM}$, inverted green triangle $=0.55 \mathrm{nM}$, purple diamond $=6.9 \mathrm{nM}$, orange circle $=55 \mathrm{nM}$ ) were spiked into: (A) QCs prepared by spiking recombinant monkey SBCMA into surrogate matrix at 75.2, 8.84, 1.04, 0.12 nM (denoted HQC, MQC, LQC, and LLOQ, respectively) and (B) four naive cynomolgus monkey plasma samples with endogenous SBCMA. As the drug concentrations increased, the recovered amount of SBCMA rapidly decreased. HQC, high quality control; LBA, ligand binding assay; LLOQ, lower limit of quantitation; LQC, low quality control; MQC, mid quality control. antibody, we developed an IA-LC-MS/MS assay using the biotinylated polyclonal goat antihuman sBCMA antibody from the DuoSet Kit and streptavidincoated magnetic beads to first isolate sBCMA from cynomolgus monkey plasma samples. Beads were washed, sBCMA eluted, and eluates were then processed to generate tryptic peptides that were analyzed by LC-MS/MS (Fig. 3A). Only one tryptic peptide, CSSTPPLTCQR, was found to have optimal properties for LC-MS/MS quantification. This peptide was confirmed to be unique to BCMA by searching the BLAST database. When $137.6 \mathrm{nM}$ of bispecific antibody was spiked results (Fig. 2B). Finding alternative reagents with distinct, nonoverlapping epitopes was likely to be difficult and timeconsuming given the small size of the shed BCMA $(\sim 6 \mathrm{kDa})$. Therefore, we turned our attention to LC-MS-based approaches that were less reliant upon reagent generation or procurement.

\section{Protein-Level IA-LC-MS/MS Assay}

Since the LBA approach was not suitable to measure total sBCMA levels in the presence of BCMA CD3 bispecific into a fixed concentration of BCMA $(10 \mathrm{nM})$ to mimic the predicted maximum concentration bispecific levels in PK/PD studies, the XIC peak area of the surrogate peptide decreased relative to the unspiked condition indicating a degree of drug interference. By titrating the capture antibody up to $15 \mu \mathrm{g}$, the drug suppression was circumvented (Fig. 3B). In the qualified assay, $20 \mu \mathrm{g}$ of capture antibody was added to each sample to ensure minimal drug interference for all expected drug concentrations. A heavy-isotope labeled version of the surrogate

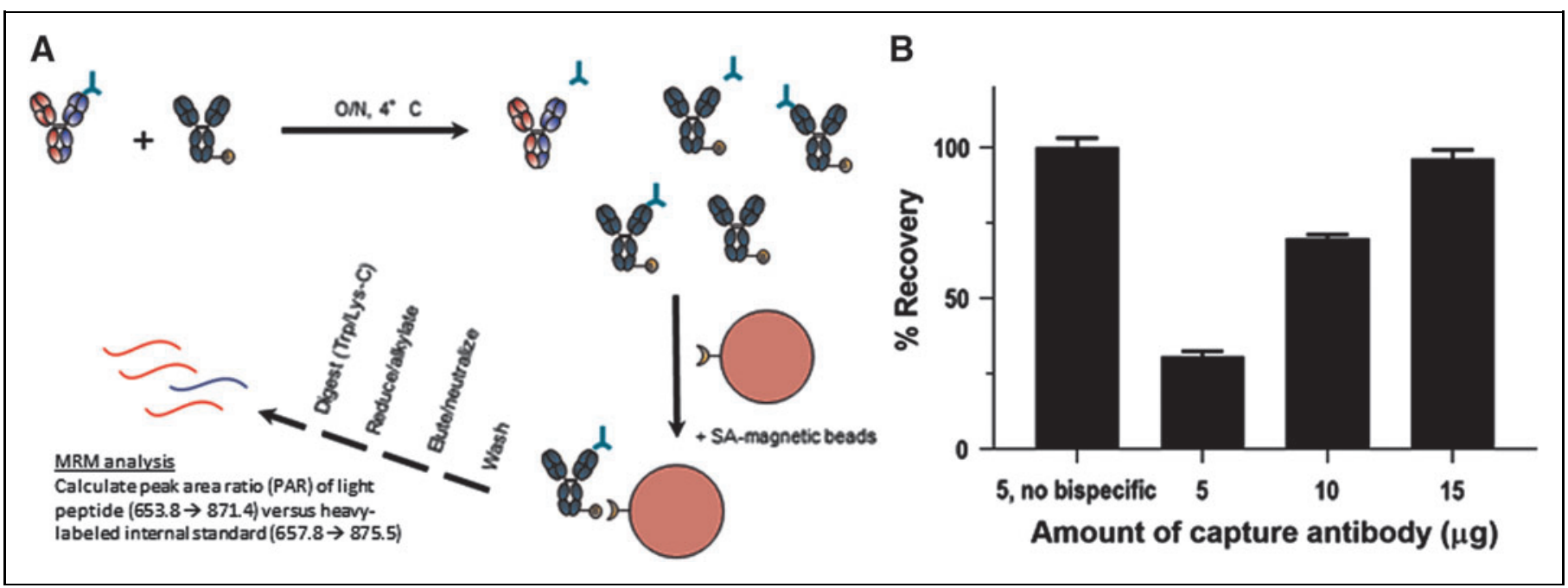

Fig. 3. Protein-level IA-LC-MS/MS workflow and minimization of drug interference. (A) SBCMA was captured with biotinylated polyclonal anti-BCMA antibodies, which were immunoprecipitated using streptavidin-coated magnetic beads. Beads were washed, bound sBCMA was eluted, and eluates were processed to generate tryptic peptides that were analyzed by LC-MS/MS. (B) Suppression of peak area in the presence of $B C M A C D_{3}$ bispecific antibody relative to the unspiked condition was overcome by titrating the capture antibody. IA, immunoaffinity; LC-MS/MS, liquid chromatography-tandem mass spectrometry. 
Table 1. Protein-Level Immunoaffinity-Liquid Chromatography-Tandem Mass Spectrometry Assay Performance Statistics for the Measurement of Soluble B Cell Maturation Antigen in Cynomolgus Monkey Plasma

\begin{tabular}{l|c|c|c|c|c|c|c|c|c} 
& Nominal concentration (nM) & Assay 1 & Assay 2 & Assay 3 & Assay 4 & Mean & SD & \% CV & \% RE \\
\hline LOC & 0.18 & 0.16 & 0.15 & 0.22 & 0.17 & 0.18 & 0.03 & 17.8 & -1.7 \\
\hline LOC + bispecific & 0.18 & 0.14 & 0.16 & 0.18 & 0.15 & 0.16 & 0.02 & 10.8 & -11.5 \\
\hline MQC & 1.2 & 1.1 & 1.1 & 1.1 & 1.1 & 1.1 & 0.04 & 3.6 & -7.7 \\
\hline HOC & 4.2 & 3.6 & 3.4 & 3.8 & 3.7 & 3.6 & 0.15 & 4.0 & -13.4 \\
\hline
\end{tabular}

CV, coefficient of variation; HQC, high quality control; LOC, low quality control; MQC, mid quality control; RE, relative error; SD, standard deviation.

peptide was also used to calculate PARs and control for intraand interinjection LC-MS/MS run variability. The final fit-forpurpose method was qualified using sBCMA standards that covered the range of expected plasma concentrations while minimizing drug interference. Using a $125 \mu \mathrm{L}$ plasma sample volume, the working range of the assay was $0.08-10.0 \mathrm{nM}$. The biases of the QCs were within 25\% of nominal, even when spiked drug $(55 \mathrm{nM})$ was added to the low quality control (Table 1). The final qualification used a drug spike concentration $(55 \mathrm{nM})$ that was $2.5 \times$ less than the initial capture antibody optimization due to new information on predicted plasma drug levels.

\section{Peptide-Level IA-LC-MS/MS}

To circumvent drug interference, a peptide-level IA-LCMS/MS assay based on the protocol originally reported by Razavi et al. ${ }^{14}$ was explored. First, rabbits were inoculated with the cynomolgus sBCMA surrogate peptide to generate antipeptide antisera. Due to chemistry limitations, a serine was substituted for the second carbamidomethylated cysteine (CSSTPPLTS $Q R$ ) in the peptide used for immunizations. The peptide-level IA-LC-MS/MS method was similar to the protein-level assay shown in Figure 3A, except that digestion, reduction, and alkylation occurred before the antibody capture step. In this assay, the XIC PAR for the sBCMA surrogate peptide was comparable with or without BCMA CD3 bispecific antibody, indicating no observable drug interference (Fig. 4). This assay format was the most selective measurement in the presence of bispecific antibody, but reagent generation took 3 months, precluding its use in the fastmoving preclinical drug development phase.

Measurement of sBCMA Levels in Cynomolgus Monkeys and Human MM Samples

Based on the assay requirements, including range, drug tolerance, time for development, and transferability, the protein-level IA-LC-MS/MS assay was used to measure
sBCMA in cynomolgus monkeys and MM patients. Seven naive cynomolgus monkey plasma samples were first tested in the protein-level IA-LC-MS/MS assay to serve as a baseline. The average sBCMA concentration was $0.19( \pm 0.3) \mathrm{nM}$ (data not shown). PK/PD dose escalation studies (1, 10, and $100 \mu \mathrm{g} /$ $\mathrm{kg})$ were then carried out in cynomolgus monkeys $(n=2$ per dose group). Plasma samples were collected at various time points, and sBCMA levels were measured. sBCMA levels increased in a dose-dependent manner, as shown in Figure 5A.

Human plasma samples from late stage MM patients were tested in the protein-level IA-LC-MS/MS assay to confirm suitability for clinical samples. The assay for human samples was easily adapted from that for cynomolgus monkey since the surrogate peptide in human SBCMA only differs by the addition of one amino acid. The sample processing was identical, while the MRM transitions were changed in the MS software to account for the additional amino acid in the human

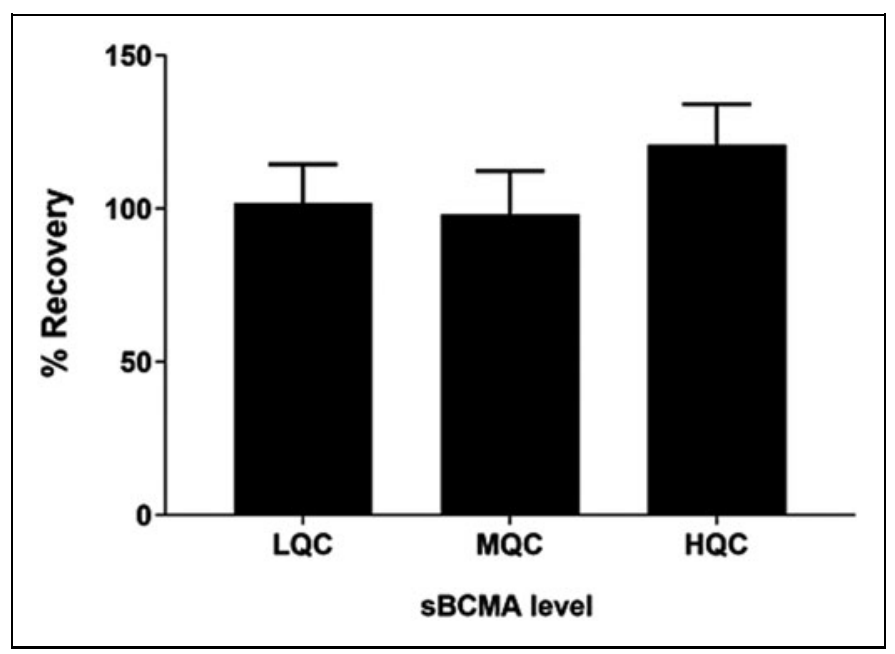

Fig. 4. Peptide-level IA-LC-MS/MS assay is not susceptible to drug interference. Peptide level IA-LC-MS/MS did not show the suppression of signal that was observed in ELISA and protein IALC-MS/MS when drug was added, indicating no drug interference in the assay. ELISA, enzyme-linked immunosorbent assay. 


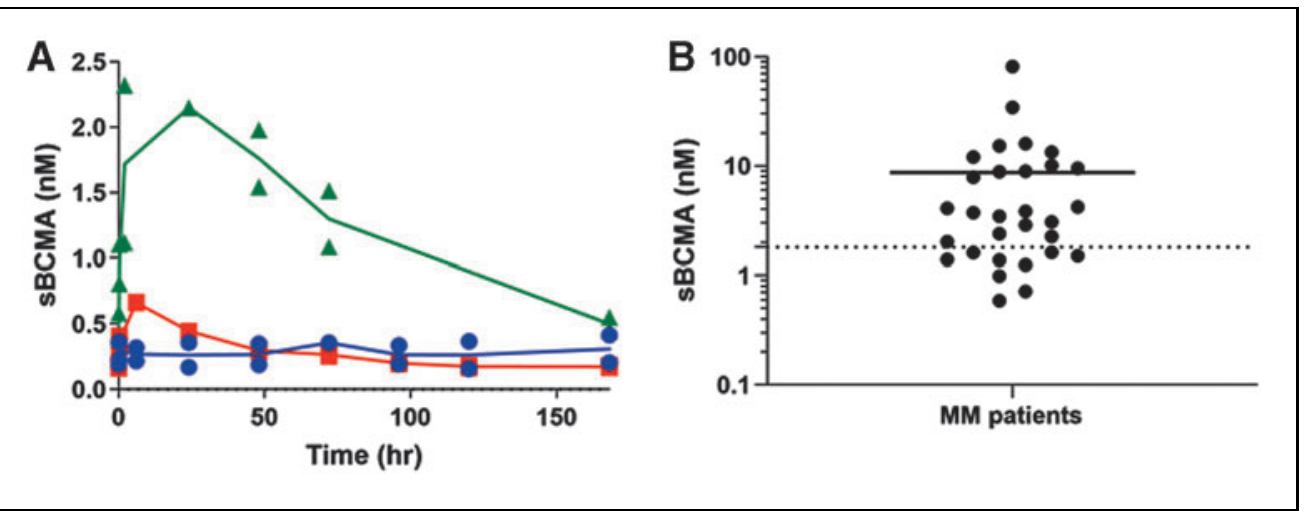

Fig. 5. Total SBCMA levels in cynomolgus monkeys dosed with bispecific antibody and in untreated human MM patients. (A) Concentration of total plasma SBCMA increases after dosing with $\mathrm{BCMA} C \mathrm{CD}_{3}$ bispecific antibody (blue circle $=1 \mu \mathrm{g} / \mathrm{kg}$, red square $=10 \mu \mathrm{g} / \mathrm{kg}$, and green triangle $=$ $100 \mu \mathrm{g} / \mathrm{kg}$ ). (B) Concentration of SBCMA in plasma samples from relapsed/refractory MM patients. SBCMA level in pooled normal plasma sample depicted by dotted line (1.75 $\mathrm{nM})$.

surrogate peptide compared to the cynomolgus monkey peptide. The mean sBCMA concentration from 30 late-stage MM patients tested was $8.7 \mathrm{nM}$, and a pooled normal human plasma sample was $1.8 \mathrm{nM}$ (Fig. 5B) ( $p=0.02$ in a one-sided $t$ test).

\section{DISCUSSION}

Three assays were assessed to determine the optimal quantitative assay for measuring total sBCMA levels in plasma. Our initial goal was to establish a fit-for-purpose assay for use in preclinical discovery cynomolgus monkey studies; however, the most desirable assay would be one that could be easily transferred to a regulated laboratory for clinical use after further optimization and validation. The modified commercial LBA Kit was straightforward to use but was not suitable for studies where animals were dosed with BCMA CD3 bispecific antibody due to competition for the binding site. The low molecular weight of sBCMA ( $\sim 6 \mathrm{kDa})$ poses significant challenges to sandwich assay LBA formats, mainly due to the fact that antibody pairs may compete for a limited number of immunogenic epitopes. Problems associated with overlapping epitopes and drug interferences can impact all immunoassay formats, highlighting the need for other approaches.

A protein-level IA-LC-MS/MS assay was developed to circumvent drug interference and epitope competition seen in the LBA. Since the IA step required use of only one anti-BCMA antibody, we theorized that drug interference would not be as much of an issue compared to that of the sandwich LBA as a result of less steric hindrance to impede analyte binding. However, drug interference was still observed. This interference was overcome in this assay format by increasing the amount of capture antibody used during immunoprecipitation. We could have potentially applied this knowledge to a different LBA format, but we chose to move forward with an MS-based assay due to inherent selectivity that results from quantification of BCMA-specific surrogate peptides by mass spectrometry. In addition, this assay had adequate sensitivity to quantitatively measure sBCMA in the concentration ranges expected in cynomolgus monkey and human samples. Furthermore, this assay format was easily adapted to measure human sBCMA with minimal methodological changes since the quantified BCMAspecific surrogate peptide differs by only one amino acid between monkey and human.

We also explored the use of a peptide-level IA-LC-MS/MS assay in lieu of the protein-level IA method, which also is inherently free of drug interference, due to initial protein digestion to peptides and elimination of all protein complexes. This method was not used for preclinical studies due to the time it took to generate polyclonal antipeptide antibodies. Another drawback of this method was that it used a considerable amount ( $100 \mu \mathrm{g})$ of antipeptide antibodies to capture the surrogate peptide out of digested matrix. This may be due to differential affinities for the substituted serine in the immunogen versus the endogenous sBCMA surrogate peptide sequence. The antipeptide approach could have been improved using an in-line antipeptide affinity column coupled with nanoflow LC systems, which offers several advantages over the antipeptide capture method in bulk digested matrix solution that was used here. These systems use minimal capture antibody to prepare a column, the columns can be reused after crosslinking, and they have the potential to reach or even surpass the sensitivity of traditional LBA methods with the added advantage of selectivity through MS/ MS analysis. ${ }^{13}$

Total sBCMA levels were measured using the protein-level IA-LC-MS/MS assay after administration of BCMA CD3 bispecific antibody to cynomolgus monkeys, and the levels increased with increasing plasma drug concentrations. This accumulation may reflect the formation of sBCMA-drug complexes, effectively extending the half-life of sBCMA, ${ }^{3}$ or from drug-induced shedding, ${ }^{1}$ although the relative contributions of these potential mechanisms were not assessed. The total sBCMA concentrations were ultimately used along with the total BCMA CD3 bispecific antibody concentrations 
and other parameters in a translational PK/PD model to predict the clinical efficacious dose in humans. By converting the IA-LC-MS/MS assay from a monkey-specific assay to a human assay, we also showed the flexibility of this assay format while retaining the selectivity inherent to MS analysis. Additional characterization of the assay is needed to validate this format for clinical testing.

Our results show that bioanalytical strategies to measure shed targets should be considered early in drug development to ensure the availability of appropriate reagents. In addition to assay parameters of required sensitivity and selectivity, the ability to measure total soluble receptor in the presence of drug must be considered. In Figure 6, we propose a decision tree for directing efforts for selecting the best assay formats with respect to minimizing drug interference. In this study, we discussed method development and considerations for measuring total soluble target, but free target measurements are sometimes considered more pharmacologically relevant. ${ }^{17}$ Free target measurements, however, present a broader range of bioanalytical challenges, including the need for very high assay sensitivity and potential overestimation of free con-

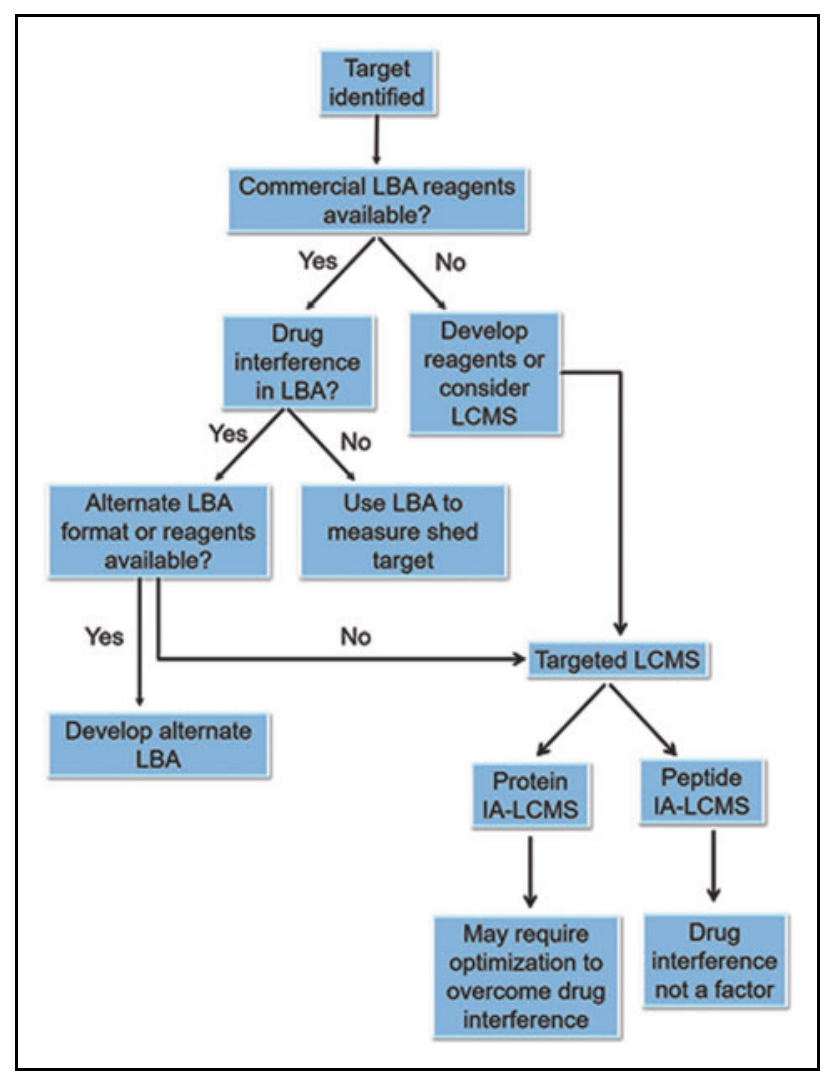

Fig. 6. Proposed decision tree for identifying best bioanalytical strategy for shed receptor analysis. centrations due to release of bound analyte upon perturbation of equilibrium during sample processing. ${ }^{17}$ Discussions regarding the need for free versus total assays should be initiated early in preclinical development and continued throughout development of the drug to ensure that the bioanalytical strategy is aligned with the needs of the project teams, including the PK/PD modeler and clinical teams.

\section{CONCLUSION}

Shedding of transmembrane proteins and release into the circulation have been reported for several biotherapeutic targets. Quantification of dynamic levels of shed target receptors may be essential for understanding the impact on drug development. In this study, we presented a bioanalytical approach to select a fit-for-purpose assay to shed BCMA levels in preclinical cynomolgus monkey PK/PD studies. This assay has been modified for analysis of human samples in preparation for use in treated MM patients. Instead of being wed to any given platform or general assay type, we propose a strategy by which multiple assay platforms are initially assessed for feasibility before comprehensive fit-for-purpose qualification of a final assay format. This strategy is enabled by housing disparate platforms in the same laboratory and a commitment to substantive cross-training of analysts. Flexibility between analytical platforms can be an asset in decreasing assay development time in ever-constrained drug development timelines. The overall bioanalytical strategy can be used as guidance for development of any shed target measurement method.

\section{ACKNOWLEDGMENTS}

The authors thank Allison Given Chunyk and Alison Betts for thoughtful discussions and Frank Barletta and Peter O'Brien for their critical review of the article.

\section{DISCLOSURE STATEMENT}

No competing financial interests exist.

\section{FUNDING INFORMATION}

No external funding for this work was received.

\section{REFERENCES}

1. Miller MA, Sullivan RJ, Lauffenburger DA: Molecular pathways: receptor ectodomain shedding in treatment, resistance, and monitoring of cancer. Clin Cancer Res 2016;23:623-629.

2. Tien WS, Chen JH, Wu KP, SheddomeDB: the ectodomain shedding database for membrane-bound shed markers. BMC Bioinformatics 2017;18(Suppl. 3): $31-48$. 
3. Samineni D, Girish S, Li C: Impact of Shed/Soluble targets on the PK/PD of approved therapeutic monoclonal antibodies. Expert Rev Clin Pharmacol 2016; 9:1557-1569.

4. Albitar M, Do KA, Johnson MM, et al.: Free circulating soluble CD52 as a tumor marker in chronic lymphocytic leukemia and its implication in therapy with anti-CD52 antibodies. Cancer 2004;101:999-1008.

5. Giles FJ, Vose JM, Do KA, et al.: Circulating CD20 and CD52 in patients with non-Hodgkin's lymphoma or Hodgkin's disease. Br J Haematol 2003;123:850857.

6. Sanchez $E, L i M$, Kitto $A$, et al.: Serum B-cell maturation antigen is elevated in multiple myeloma and correlates with disease status and survival. $\mathrm{Br} J$ Haematol 2012;158:727-738.

7. Laurent $\mathrm{SA}$, Hoffman FS, Kuhn PH, et al:: Gamma-secretase directly sheds the survival receptor BCMA from plasma cells. Nat Commun 2015;6:73337345.

8. Lee JW, Kelley $M$, King $L E$, et al:: Bioanalytical approaches to quantify "total" and "free" therapeutic antibodies and their targets: technical challenges and PK/PD applications over the course of drug development. AAPS J 2011;13: 99-110.

9. Ahene $A B$ : Application and interpretation of free and total drug measurements in the development of biologics. Bioanalysis 2011;3:12871295.

10. Patton A, Mullenix MC, Swanson SJ, et al:: An acid dissociation bridging ELISA for detection of antibodies. J Immunol Methods 2005;304:189-195.

11. Verch $T$, Chilewski $S$, Bouaraphan $S$, et al:: Pharmacokinetic immunoassay methods in the presence of soluble target. J Immunol Methods 2010;361: 75-81.

12. Chappell DL, Lassman ME, McAvoy T, et al.: Quantitation of human peptides and proteins via MS: review of analytically validated assays. Bioanalysis 2014;6: 1843-1857.

13. Neubert $H$, Gale J, Muirhead D: Online high-flow peptide immunoaffinity enrichment and nanoflow LC-MS/MS: assay development for total salivary pepsin pepsinogen. Clin Chem 2010;56:1413-1423.

14. Razavi M, Anderson NL, Pope ME, et al.: High precision quantification of human plasma proteins using the automated SISCAPA Immuno-MS workflow. N Biotechnol 2016;33:494-502.

15. Hipp S, Tai Y-T, Blanset $D$, et al:: A novel BCMA/CD3 bispecific T-cell engager for the treatment of multiple myeloma induces selective lysis in vitro. Leukemia 2017:31:1743-1751.

16. Seckinger $A$, Delgado JA, Moser $S$, et al:: Target expression, generation, preclinical activity, and pharmacokinetics of the BCMA-T cell bispecific antibody EM801 for multiple myeloma treatment. Cancer Cell 2017;31:396410.

17. Talbot JJ, Calamba D, Pai $M_{\text {, et }}$ al:: Measurement of free versus total monoclonal antibody in pharmacokinetic assessment is modulated by affinity, incubation time, and bioanalytical platform. AAPS J 2015;17:14461454
Address correspondence to:

Michael Hall, PhD

Biomedicine Design

Pfizer Worldwide Research, Development, and Medical

10646 Science Center Drive

San Diego, CA 92121

USA

E-mail: michael.hall@pfizer.com

\section{Abbreviations Used}

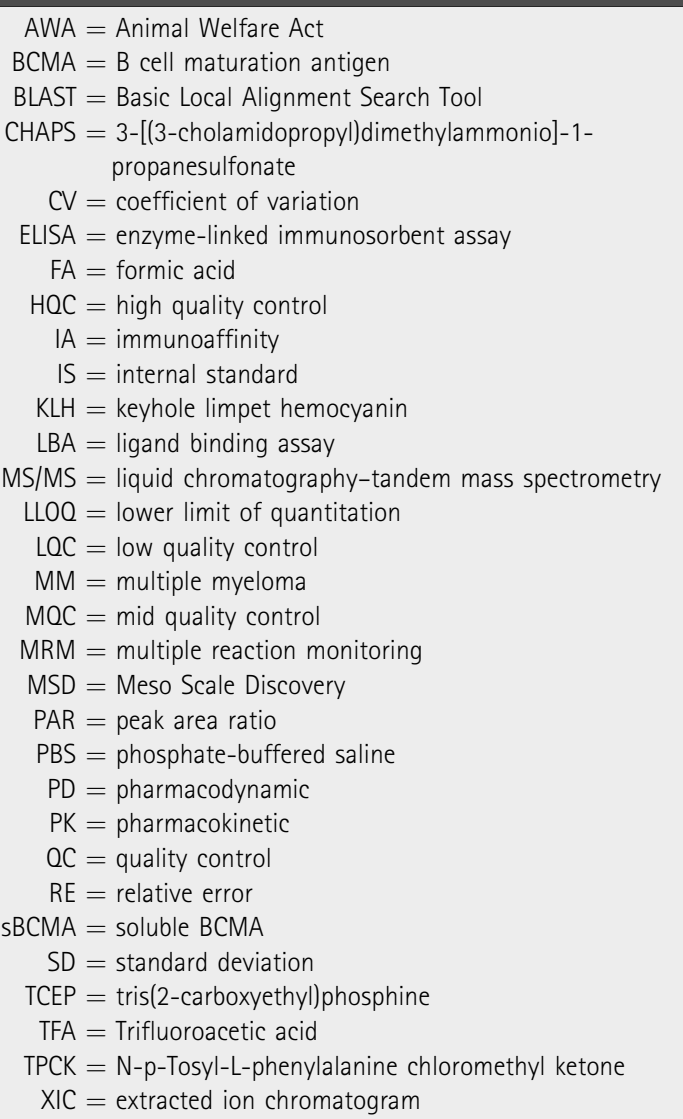

